\title{
Neovascularization of hepatocellular carcinoma in a nude mouse orthotopic liver cancer model: a morphological study using X-ray in-line phase-contrast imaging
}

Beilei $\mathrm{Li}^{1,2+}$, Yiqiu Zhang ${ }^{1,2+}$, Weizhong $\mathrm{Wu}^{3}$, Guohao Du ${ }^{4}$, Liang Cai ${ }^{1,2}$, Hongcheng Shi ${ }^{1,2}$ and Shaoliang Chen ${ }^{1,2^{*}}$

\begin{abstract}
Background: This study aimed to determine whether synchrotron radiation (SR)-based X-ray in-line phase-contrast imaging (IL-PCl) can be used to investigate the morphological characteristics of tumor neovascularization in a liver xenograft animal model.

Methods: A human hepatocellular carcinoma HCCLM3 xenograft model was established in nude mice. Xenografts were sampled each week for 4 weeks and fixed to analyze tissue characteristics and neovascularization using SR-based X-ray in-line phase contrast computed tomography (IL-XPCT) without any contrast agent.

Results: The effect of the energy level and object-to-detector distance on phase-contrast difference was in good agreement with the theory of IL-PCI. Boundaries between the tumor and adjacent normal tissues at week 1 were clearly observed in two-dimensional phase contrast projection imaging. A quantitative contrast difference was observed from weeks 1 to 4 . Moreover, 3D image reconstruction of hepatocellular carcinoma (HCC) samples showed blood vessels inside the tumor were abnormal. The smallest blood vessels measured approximately $20 \mu \mathrm{m}$ in diameter. The tumor vascular density initially increased and then decreased gradually over time. The maximum tumor vascular density was $4.29 \%$ at week 2.
\end{abstract}

Conclusion: IL-XPCT successfully acquired images of neovascularization in HCC xenografts in nude mice.

Keywords: Synchrotron radiation, In-line phase-contrast imaging, Computed tomography, Hepatocellular carcinoma, Tumor neovascularization

\section{Background}

Neovascularization is an important feature of solid tumors [1]. Evaluation of tumor neovascularization is helpful for tumor diagnosis, prognosis and assessment of anti-angiogenic efficacy. Vascular imaging techniques including computed tomography angiography (CTA), magnetic resonance angiography (MRA) and digital subtraction angiography (DSA) are based on the differences in the vascular structures and blood flow between tumor and normal vessels, and have been used to monitor

\footnotetext{
* Correspondence: csl20150507@126.com

${ }^{\dagger}$ Equal contributors

'Department of Nuclear Medicine, Zhongshan Hospital, Fudan University, No.180, Fenglin Road, Shanghai 200032, China

${ }^{2}$ Shanghai Institute of Medical Imaging, Shanghai 200032, China

Full list of author information is available at the end of the article
}

tumor angiogenesis or determine the efficacy of antiangiogenic therapies. However the spatial resolution, especially when detecting small vessels with a diameter of less than $200 \mu \mathrm{m}$ is still limited [2,3]. Even microCTA, which has the highest resolution among these methods, can only observe vessels of no less than $50 \mu \mathrm{m}$ in diameter $[4,5]$.

Synchrotron radiation (SR) microvascular angiography combined with high-resolution and high-speed imaging systems provide an effective approach to study tumor angiogenesis. In vitro studies at the SPring-8 BL20B2 facility in Japan, using barium sulfate as a contrast agent, have revealed the micro-vessel architecture of VX2 carcinoma specimens [6]. Using iodine as contrast agent, SR micro-angiography reliably detects tumor micro- 
vessel density in vivo [7]. Neovascularization in Lewis lung cancer tumor located deep inside the body was observed using a three-dimensional reconstruction of micro-CT imaging with barium sulfate as contrast agent [8]. However, all these studies used absorption contrast between the contrast medium and surrounding tissues.

Different from these attenuation-based X-ray imaging, $\mathrm{X}$-ray phase-contrast imaging (PCI) exploits differences in the refractive index of different materials to differentiate structures [9]. Thus, PCI can clearly define weakly $\mathrm{X}$-ray-absorptive biological soft tissues without the use of a contrast agent. Several PCI modalities have been developed, such as interferometry [10], diffraction enhanced imaging (DEI) [11, 12], grating-based phasecontrast X-ray imaging (GB-PCI) [13], and in-line phase contrast imaging (IL-PCI) $[14,15]$. Among them, IL-PCI has no requirements of superior temporal coherence within the X-ray source and complex experimental apparatus in the light path. Synchrotron-based X-ray Tomographic Microscopy (SRXTM) has been described as a powerful technique for non-destructive highresolution investigations of various materials, allowing micrometer and sub-micrometer, quantitative, threedimensional imaging; other techniques include the Swiss Light Source TOMCAT, a new beamline for Tomographic Microscopy and Coherent radiology experiments, which offers sensitivity to density differentials within soft tissues and permits the accommodation of larger tissue sizes $[16,17]$. Although studies are currently limited to the stage of technique optimization with in vitro specimen analysis, SR-based X-ray in-line phase contrast computed tomography (IL-XPCT) has great potential for future clinical diagnostic application.

Using the third-generation synchrotron radiation light source at the Shanghai Synchrotron Radiation Facility (SSRF), IL-XPCT has achieved good results in microvascular and tumor angiogenesis $[18,19]$. The aim of the present study was to investigate the morphological characteristics of tumor neovascularization in a human hepatocellular carcinoma (HCC) xenograft model using SR-based IL-XPCT.

\section{Methods}

\section{Animals}

Male BALB/c athymic nude mice (5-6 weeks old, weighing $15-18 \mathrm{~g}$ ) were purchased from SLAC Laboratory Animal Co., Ltd. (Shanghai, China), and maintained under specific pathogen-free (SPF) conditions at the Animal Center of the Liver Cancer Institute of Zhongshan Hospital affiliated to Fudan University (Shanghai, China). All animal experiments were approved by the Animal Care and Use Committee of Zhongshan Hospital, and were conducted in accordance with all state regulations.

\section{Cell culture}

The HCCLM3 cell line was established by the Liver Cancer Institute, Zhongshan Hospital, Fudan University, China, as a HCC cell line with high metastatic potential. Cells were maintained in high-glucose Dulbecco's modified eagle medium (D-MEM; GibcoBRL, Grand Island, New York, USA) supplemented with $10 \%$ fetal bovine serum (Hyclone, Utah, USA) in a humidified $5 \% \mathrm{CO}_{2}$ atmosphere at $37^{\circ} \mathrm{C}$.

\section{Orthotopic xenograft model}

A single mouse was subcutaneously injected with $1 \times$ $10^{7} / 0.2 \mathrm{ml}$ HCCLM3 cells in the right upper flank region for the establishment of a subcutaneous xenograft model. When the subcutaneous tumor reached $1 \mathrm{~cm}$ in diameter (approximately 4 weeks after injection), it was removed, cut into small pieces of equal volume $\left(1 \mathrm{~mm}^{3}\right)$, and transplanted into the left lobe of the liver of 24 nude mice to establish orthotopic xenograft models, as previously described [20].

\section{Preparation of liver samples}

Each week after grafting, six nude mice were anesthetized by intraperitoneal injection of sodium pentobarbital $(0.016 \mathrm{~g} / \mathrm{mL}, 0.5 \mathrm{~mL} / 100 \mathrm{~g})$. After opening the abdominal cavity, a PE 10 catheter (Smiths Medical, London, UK) was inserted into the inferior vena cava to inject heparinized saline. When the liver looked pale, the blood vessels and bile ducts were ligated, and the liver was resected. Specimens were immersed in $4 \%$ formaldehyde for tissue fixation at room temperature overnight. The next day, three samples were washed and dehydrated using graded ethanol for IL-PCI. Three other samples were used for immunohistochemistry.

\section{X-ray IL-PCl settings}

Neovascularization imaging of tumor xenografts was performed at the X-ray imaging and biomedical application beamline (BL13W1) of the SSRF. The experimental set-up is shown in Fig. 1. The SSRF BL13W1 imaging device was a third-generation synchrotron source with a $200 \mathrm{~mA}$ beam current and $3.5 \mathrm{GeV}$ storage energy. The $\mathrm{X}$-ray flux of BL13W1 was several orders of magnitude of X-ray tube flux; the device was designed to provide photon energy ranging from 8 to $72.5 \mathrm{keV}$ with a beam size of $48 \mathrm{~mm}$ (horizontal) $\times 5 \mathrm{~mm}$ (vertical) at the object position at $20 \mathrm{keV}$. Objects are placed at approximately $34 \mathrm{~m}$ from the source (storage ring), and the detector can be placed at 0 to $8 \mathrm{~m}$ from the objects. Based on the sample size, a high resolution detector VHR 1:1 (Photonic Science, Roberts Bridge, East Sussex, UK) was used with an effective pixel size of $9 \mu \mathrm{m}$. Because energy, distance and image quality are not linearly correlated, we used different X-ray energies $(12,15$ and $20 \mathrm{keV})$ and object-to-detector distances $(0.05,1,3$ and $5 \mathrm{~m})$. The 


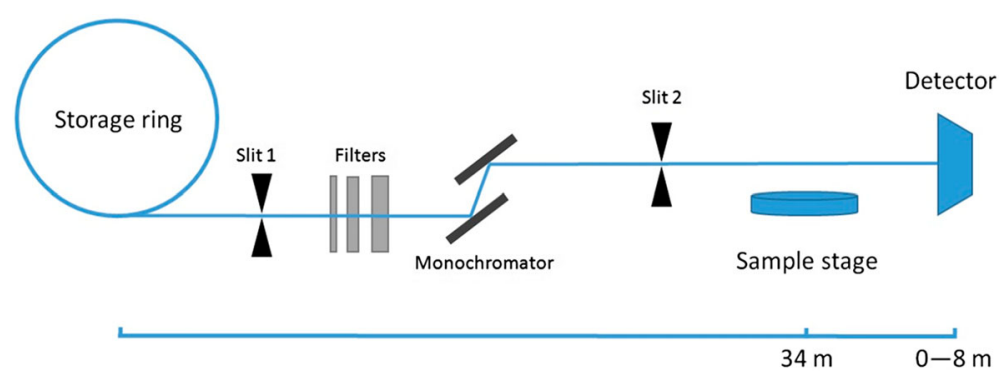

Fig. 1 The SSRF BL13W1 imaging device schematic diagram

optimal X-ray energy and object-to-detector distance were selected and used in subsequent experiments.

For the acquisition of $\mathrm{CT}$ images, the sample was rotated $180^{\circ}$ at a speed of $0.25^{\circ} / \mathrm{s}$, for a total of 1200 projection images. The exposure time of each projection image was $2 \mathrm{~s}$. All projection images were transformed into digital slice sections using the fast slice reconstruction software (compiled by the BL13W1 experimental station) based on the filtered back projection (FBP) algorithm. Threedimensional reconstruction was obtained using the VG
Studio Max 3D reconstruction software (version 2.1, Volume Graphics GmbH, Germany).

\section{Image analysis}

Phase contrast image evaluation

A normal hepatic lobe was taken for imaging at different X-ray energy levels and object-to-detector distances. Four regions of interest (ROIs) and two internal vessels (Fig. 2a) were selected in each frame to calculate
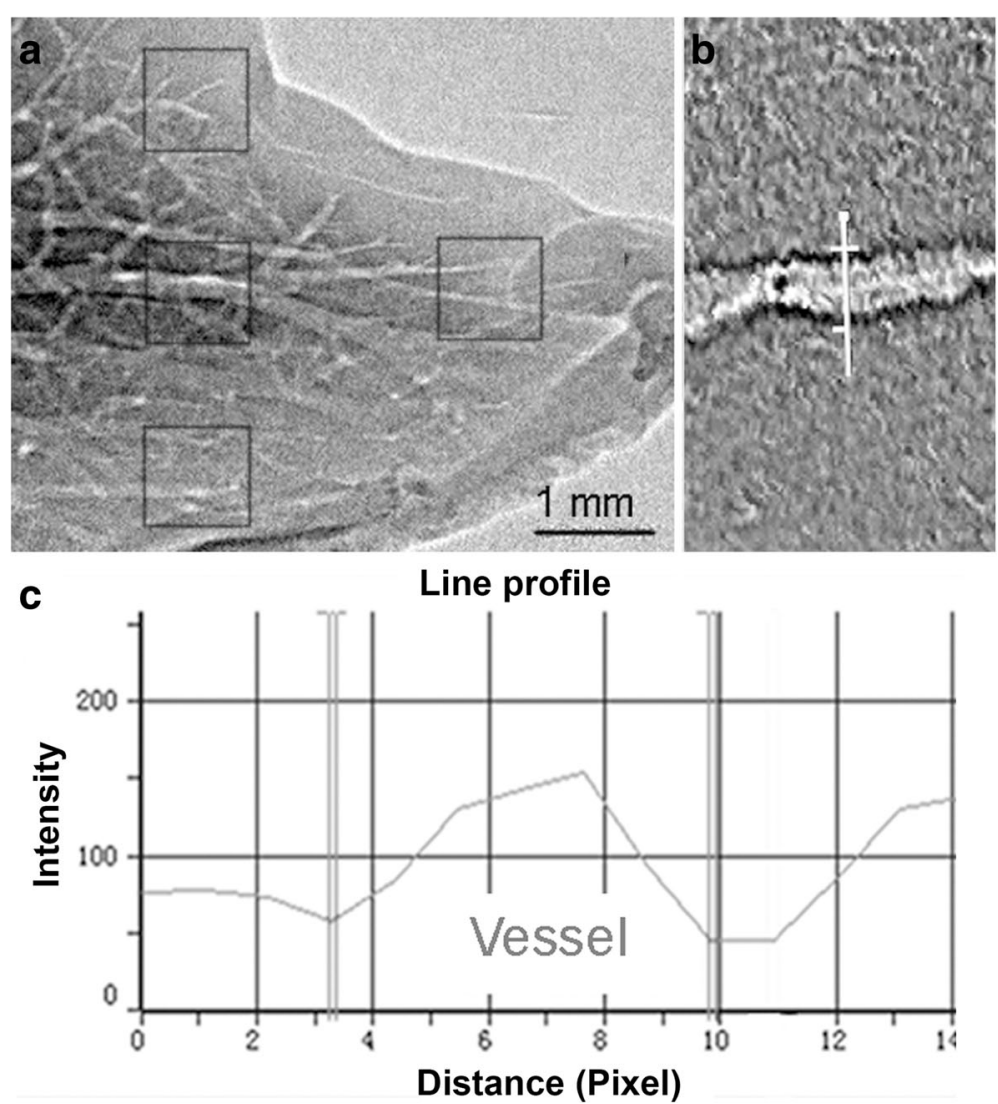

Fig. 2 Method for calculating image contrast. a Four ROls were labeled, and the two internal vessels were selected for calculating image contrast. Vessel boundaries were identified using a computer software (Image Pro Plus 6.0) that can identify both edges of a vessel (b) by expressing a density curve with a 256 gray-scale image (c) 
the image contrast according to the following formula $[8,21]$ :

$$
C=\frac{I_{\max }-I_{\min }}{I_{\max }+I_{\min }}
$$

in which $I_{\max }$ and $I_{\min }$ represent the gray scale values on either side of the blood vessel wall as determined using the Image Pro Plus 6.0 software (Media Cybernetics Inc., Rockville, MD, USA; Fig. 2b, c).

\section{Quantification of tumor neovascularization using two- dimensional phase-contrast projection}

Compared with surrounding normal liver tissues, the tumor vessels are relatively smaller, with a low density, resulting in different vascular boundary enhancements in two-dimensional projection images. Based on this principle, a two-dimensional phase-contrast image projection at different tumor growth stages was analyzed using the Analyze 10.0 software (Analyze Direct, Inc., Lenexa, KS, USA). First, a threshold method was used to correct the area with transmittance below a given value to eliminate the impact of the suture inside the tumor. To quantify the fluctuation of image intensities formed in the detector plane, we used the following equation to calculate the contrast projection, which is composed of overlapping local contrasts [22]:

$$
C(x, y)=\frac{\sqrt{<I(x, y)^{2}>_{W}-<I(x, y)>_{W}^{2}}}{<I(x, y)>_{W}}
$$

In this formula, $(x, y)$ represents a point in the image, $I$ is the intensity; subscript $W$ denotes the size of the local calculation window, and the operator $<">$ signifies averaging over the local window.

\section{Quantitative analysis of tumor neovascularization using SR-based IL-XPCT}

Tomographic images at different tumor growth stages were analyzed using the Analyze 10.0 software. The tumor was segmented manually in the tomographic image to calculate tumor volume $\left(\mathrm{mm}^{3}\right)$. A threshold method was used to extract vessels from large quantities of data. The smallest tumor vascular diameter $(\mu \mathrm{m})$, tumor neovascularization volume $\left(\mathrm{mm}^{3}\right)$ and vascular density (tumor neovascularization volume/tumor volume) were assessed [23].

\section{Immunohistochemistry analysis of microvessel density}

Tumor specimens were fixed in $4 \%$ formalin, embedded in paraffin, and sliced into $4-\mu \mathrm{m}$-thick serial sections. Slices were analyzed by hematoxylin-eosin (H\&E) staining. Microvessel density (MVD) was determined by immunohistochemistry using an anti-CD34 antibody (C-18,
Santa Cruz Biotechnology Inc., USA). The number of vessels was scored using a previously described method [24] under a light microscope at $200 \times$ magnification. Any single or cluster of cells with brown staining and clearly separated from adjacent microvessels, tumor cells, and other connective-tissue elements were counted as a single microvessel.

\section{Statistical analysis}

Quantitative data were presented as mean \pm standard deviation (SD), except for MVD (non-normal distribution), which was expressed as median (interquartile range [IQR]). Normally distributed data were analyzed by repeated measures analysis of variance (ANOVA), with post hoc Bonferroni t-tests. Simple linear regression models were used to assess the trend of the changes of tumor development by analyzing tumorand vascular volumes $\left(\mathrm{mm}^{3}\right)$ in relation to time. For MVD, differences between samples were analyzed using the Kruskal-Wallis test. All $P$-values were two sided, and $P<0.05$ was considered statistically significant. Data were analyzed using SAS 9.2 (SAS Institute, Inc., Cary, NY, USA).

\section{Results}

\section{Selection of imaging conditions}

We first tested result quality using a normal liver sample. Figure 3a shows a normal liver lobe imaged at different X-ray energy levels and object-to-detector distances. Using an object-to-detector distance of $0.05 \mathrm{~m}$, only large branching vessels were visible with low contrast, and the liver boundaries were obscure. At $15 \mathrm{keV}$, when the distance was increased to $1 \mathrm{~m}$, the progressive branching of the liver vessels was visible, and the tiny vessels at the outer edge of the liver lobe were clearly displayed. However, images were slightly blurry when the distance was increased from 3 to $5 \mathrm{~m}$. Therefore, the X-ray energy was set to $15 \mathrm{keV}$ and the object-todetector distance at $1 \mathrm{~m}$ for the subsequent experiments (Fig. 3b).

\section{Tumor neovascularization using X-ray IL-PCI}

The two-dimensional phase contrast projection imaging showed that the normal liver vascular structures were occupied by the tumor tissue (Fig. 4, 1w-a, 1w-b, 2w-a, $2 \mathrm{w}-\mathrm{b}, 3 \mathrm{w}-\mathrm{a}$ and $4 \mathrm{w}-\mathrm{a})$, with clear boundaries between tumor and non-tumor tissues. Along with an increased tumor volume, the tumor margin and peripheral vasculature were under increased pressure, showing tissue compression. The distribution of blood vessels within the tumor was disorganized and had an irregular appearance. In addition, the tumor presented a lobulated shape as the tumor volume increased. 


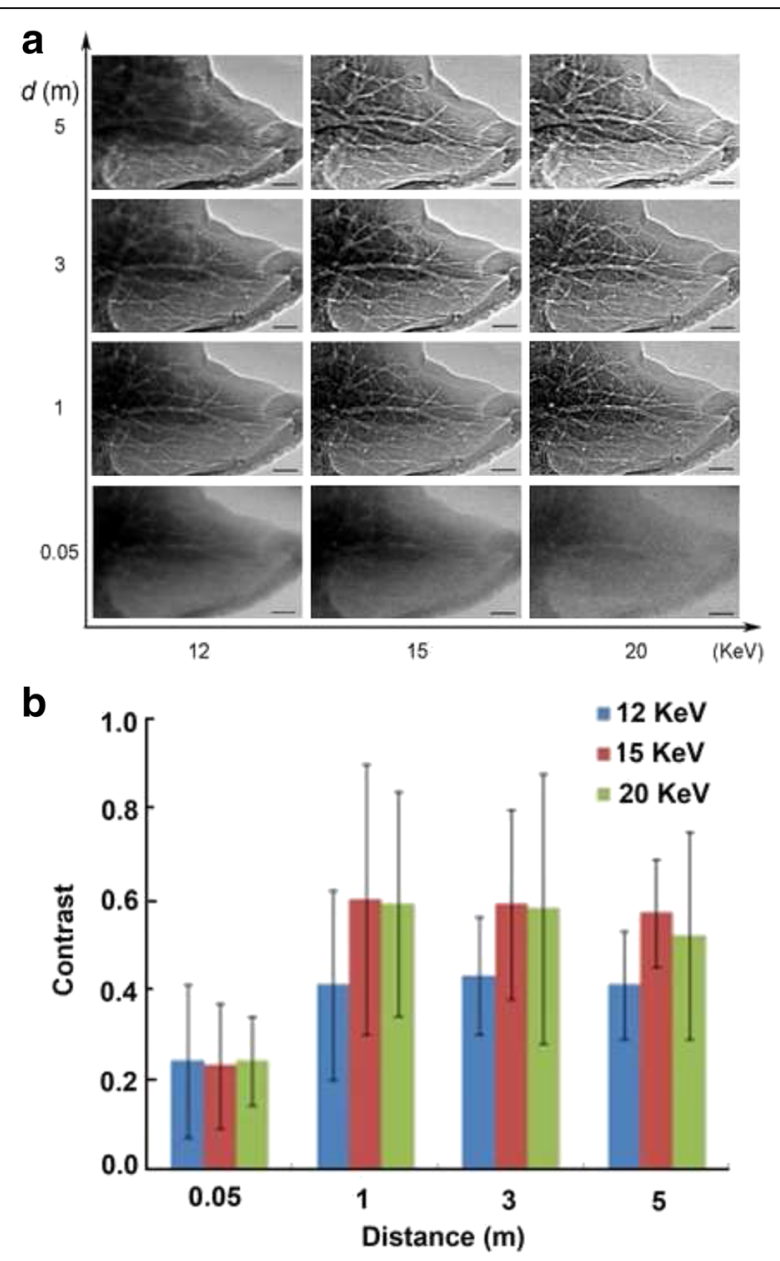

Fig. 3 Two-dimensional projection images vs. hepatic vascular phase contrast at different $\mathrm{X}$-ray energies and object-to-detector distances. a Normal liver lobe with X-rays set to 12, 15 and $20 \mathrm{keV}$. Each energy condition was used at 0.05, 1, 3 and $5 \mathrm{~m}$. Bar $=1 \mathrm{~mm}$. b Quantitative comparison of image contrast at different X-ray energy levels and object-todetector distances. The best image contrast was obtained using $15 \mathrm{keV}$ and at $1 \mathrm{~m}$

Quantification analysis of the X-ray phase contrast images (Fig. 4, 1w-a, 1w-b, 2w-a, 2w-b, 3w-a and 4w-a) using Eq. (2) showed significant differences in vascular boundary enhancement effects at weeks 1 to 4 , which are represented by a higher contrast in the normal liver tissue, and a relatively low contrast in tumor tissue (Fig. 4,1w-c, 2w-c, 3w-b and 4w-b). Along with increasing tumor volume, a large number of vessels at the tumor margin were compressed. The vascular boundary enhancement resulted in higher contrast (Fig. 4,4w-b). It is noteworthy that the normal liver tissue presented as a thin edge. Vascular boundary enhancement was lower in these regions compared with thicker liver tissues near the porta hepatis or close to liver tumor tissues, resulting in lower contrast (Fig. 4,3w-b and 4w-b).
Figure 5 presents a three-dimensional structural reconstruction of tumors at different growth stages. There was much neovascularization at weeks 1 and 2 (Fig. 5a and $b$ ), but the avascular regions gradually increased thereafter (Fig. 5c and d). Vessels had an irregular shape with partially visible dendritic branching (Fig. 5e). There were abnormal curvatures of individual vessels, with both large and small curvatures (Fig. 5f). A vessel network cluster structure was seen within the tumor at weeks 3 and 4 (Fig. $5 \mathrm{~g}$ ). A large number of curved tiny blood vessels branched from several large vessels (Fig. 5h). Finally, tumor edge or peripheral vessels were compressed, and presented as having arcuate displacement (Fig. 5c, d).

Table 1 shows tumor volume, vascular volume and vascular density at weeks 1 to 4 . Tumor volume and vascular volume increased with time, but the changes in tumor volume were much greater than those in tumor vascular volume. Although the number of new vessels increased gradually, the tumor growth rate was greater than the angiogenetic. Therefore, vascular density first increased and then decreased during growth. At week 2, tumors had the highest vascular density $4.29 \pm$ $0.49 \%$. The smallest blood vessels measured in SR images were approximately $20 \mu \mathrm{m}$ in diameter. At different stages of tumor growth, vessels of 27 to $54 \mu \mathrm{m}$ in diameter had the highest density.

We used gray scale analysis to monitor the influence of ring artifact (Fig. 6). The gray intensity difference between the vessels and ring artifacts were close, and there were no significant differences in phase contrast CT. Therefore, the ring artifacts had a significant impact on vascular identification during the vessel extraction process.

\section{Micro-vessel density by immunohistochemistry}

$H \& E$ staining and CD34 immunohistochemistry results are shown in Fig. 7. The boundaries between tumor and normal liver tissue could be shown clearly (Fig. 7a and b, white arrows). CD34 expression was positive in the abundant normal hepatic sinusoid (Fig. 7c and d, black asterisks) and tumor angiogenesis (Fig. 7c to f, black arrows and arrow heads). Tumor edge or peripheral vasculature was compressed. Distribution of angiogenesis was disordered within the tumor. The micro vascular-rich regions were diffusely distributed at the edge of tumor nests. Neovascularization with different diameters was seen, and abnormal large vessels (Fig. 7e and f, black arrow heads) and slit-like small vessels (Fig. 7c to f, black arrows) coexisted. The characteristics of vascular arrangements in PCI images were partly similar with that of histological sections. In addition, necrosis could be seen in tumor nests (Fig. 7c and d, white asterisks). 

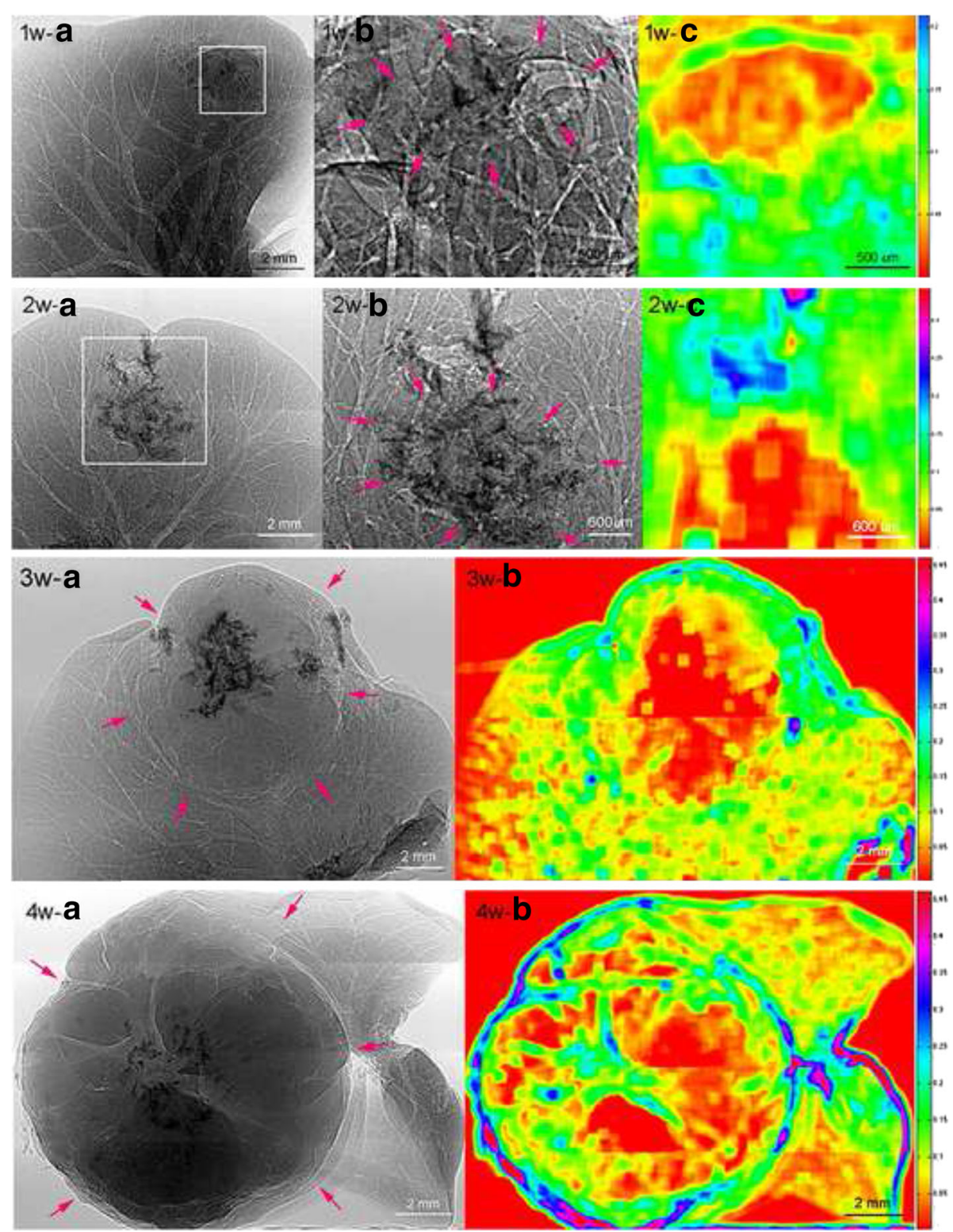

Fig. 4 Two-dimensional phase contrast projection imaging of HCCLM3 liver xenografts at weeks 1 to 4 . $1 w$-b and $2 w$-b are magnified images of the boxed regions in $1 w-a$ and $2 w-a$, respectively. The red arrows indicate the margins of the tumor, in which the normal hepatic vascular structure was damaged and replaced. The dark region is the shadow from the suture. The vascular boundary enhancement in the tumor region was detected as low contrast in the quantitative analysis results ( $1 w-c, 2 w-c, 3 w-b, 4 w-b)$. Normal liver tissue presented as a thin edge with low contrast, close to the tumor tissue $(3 w-b, 4 w-b)$

The MVD based on CD34 expression at weeks 1 to 4 was 25 (12-35) vessels/high-powered field (HPF), 16.5 (15-20) vessels/HPF, 29 (16-40) vessels/HPF, and 20 (15-25) vessels/HPF, respectively. There were no significant differences in MVD values between time points $(P=0.758)$.

\section{Discussion}

Neovascularization reflects tumor behavior and characteristics, and has received much attention in recent years
[25, 26]. PCI displays a high sensitivity for soft tissues such as blood vessels $[4,15,19,27,28]$. In the present study, the neovascularization of transplanted HCCLM3 tumors was imaged at different growth stages, and morphology and spatial distribution of tumor angiogenesis were observed.

For IL-PCI, the X-ray energy and the distance from object to detector are two important parameters $[8,9,29]$. Indeed, the lower the energy, the higher the contrast. However, low energy reduces X-ray penetration, extends 

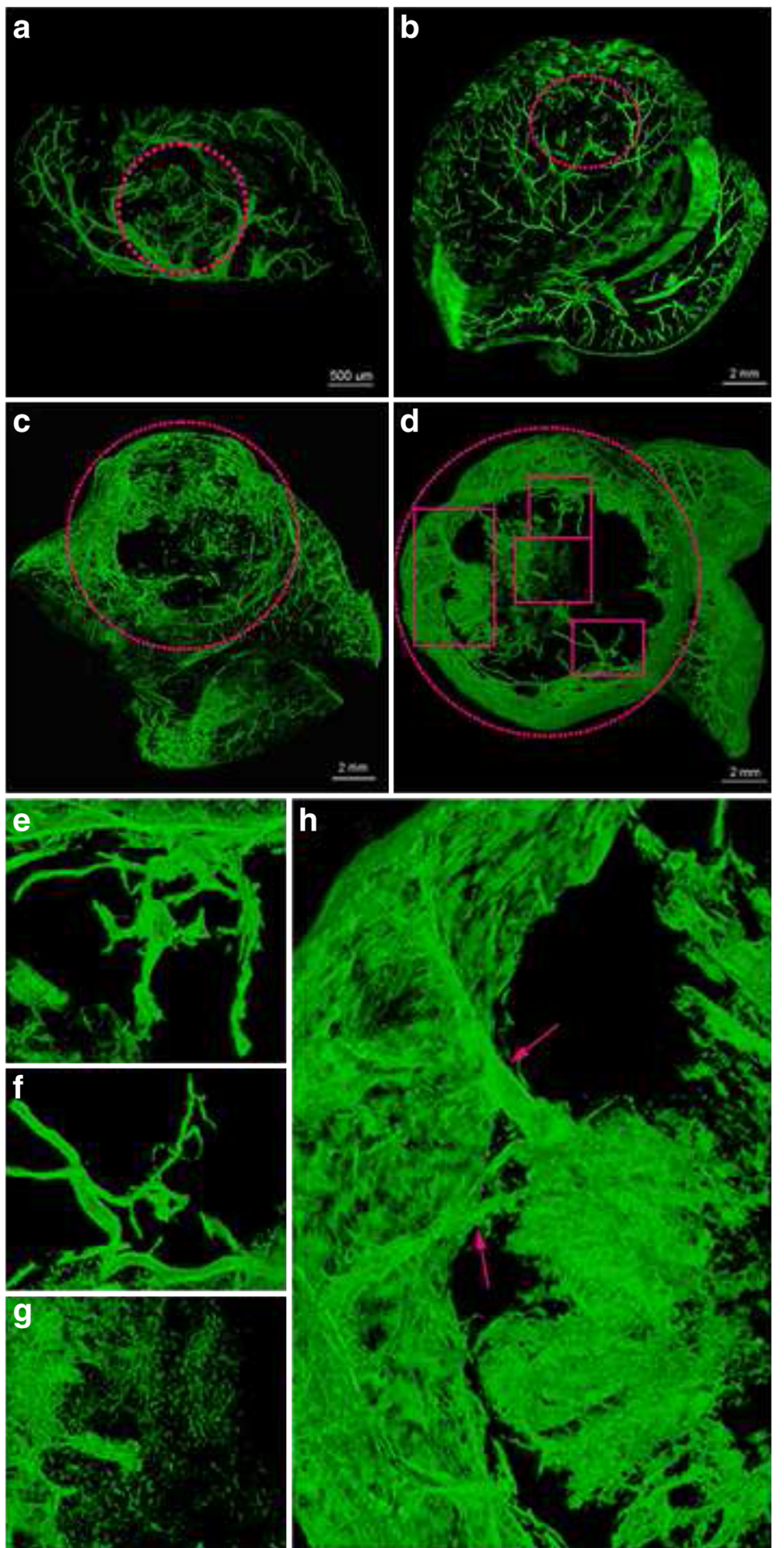

Fig. 5 3D reconstruction of contrast CT images for HCC neovascularization at weeks 1 to 4 (a to d, respectively). The selected areas with red dot lines show the tumor regions, (e to $\mathbf{f}$ ) enlarged image of the rectangle region of $d$, which clearly shows the disorganized distribution and morphology of tumor neovascularization. A large number of avascular regions were observed in the tumors at weeks 3 and 4 (c, $\mathbf{d}$ ), as well as irregular vessel shape with dendritic-like branching $(\mathbf{e})$, individual vascular curvature abnormalities $(\mathbf{f})$, blood vessel network cluster structure $(\mathbf{g})$, a large number of tiny and curved vessels derived from a few thick vessels (h, red arrows), and compressed tumor edge or peripheral vasculature (c, d) 
Table 1 Characteristics of the HCCLM3 liver xenografts at different time points

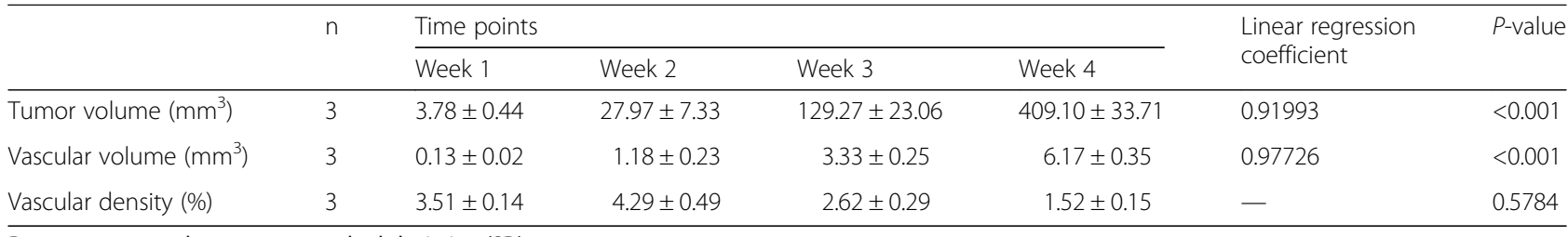

Data are presented as mean \pm standard deviation (SD)

imaging time, and increases the radiation dose. Thus, the energy level should be selected according to the specific target object in order to balance contrast and radiation dose. Based on Fresnel's diffraction theory of wave optics, the object-to-detector distance is zero in traditional absorption imaging. However, phase-contrast images are formed with greater distances from the object. The visual appearance of phase-contrast enhancement in the final image is the edge enhancement at interfaces between components with differing $\mathrm{X}$-ray refraction indices. Increasing object-to-detector distance leads to phase contrast enhancement, which is affected by the interference between the sample and X-rays; however, a distance beyond a certain range causes decreased spatial resolution, with the image losing resemblance [9]. Also, PCI details increase with time, because prolonged exposure to air and heat from the X-rays gradually dehydrates the samples $[21,30]$. In addition, specimen thickness, the fixation method, and exposure time are important factors affecting image quality $[21,30]$. According to the sample size and thickness, a CCD detector with a resolution of $9 \mu \mathrm{m}$ was selected, the X-ray energy was set to $15 \mathrm{keV}$, and the distance from source to detector was $1 \mathrm{~m}$. Based on the principle that the image occupies the whole dynamic range without saturation, we selected the appropriate exposure time and obtained a satisfactory edge enhancement effect using IL-PCI.

Based on the differences in vascular edge enhancement between tumors and adjacent normal liver tissues, quantitative analysis of the X-ray phase contrast images showed a higher contrast in the normal liver tissue, and a relatively lower contrast in tumor tissues. However, the calculation method used in this study is affected by many factors, including tissue thickness and the signalto-noise ratio [22].

IL-XPCT is suitable for the detailed 3D morphological imaging of small structure [27, 31-33]. As shown above, differences were found in tumor vascular density at different growth stages. Tumor vascular density first increased and then decreased over time. This may be related to the highly malignant ability of the HCCLM3 cell line used in the present study. At the late stage of tumor growth, tumor cells multiply quickly, leading to inadequate nutrient supply and abundant tumor necrosis. In addition, the smallest distinguishable tumor vessel diameter was found to be $20 \mu \mathrm{m}$.

Currently, immunohistochemistry is considered the "gold standard" for measuring MVD [24]. However, no significant difference in MVD was found in this study during the four weeks; this may be due to the small sample size. In addition, immunohistochemistry only reveals the two-dimensional distribution of blood vessels, and the observer selectively counts the tumor vessels in the "hot zone". Therefore, immunohistochemistry may not reflect the morphology and spatial distribution of tumor angiogenesis, resulting in the loss of potentially critical information about the vascular structure.
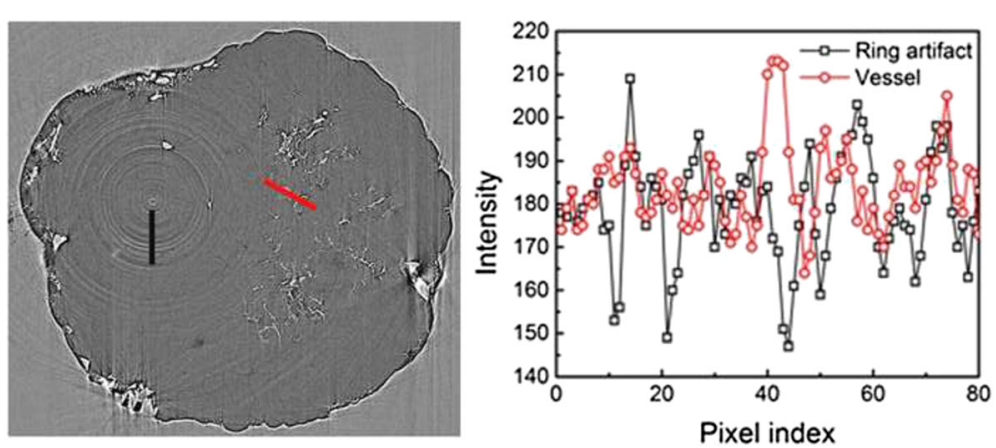

Fig. 6 Gray scale analysis of vessels and ring artifacts using two-dimensional PCI tomographic imaging. Left: The gray scale analytical measurement ranges of vessels and ring artifacts is indicated in red and black. Right: The vertical axis in the graph is gray intensity, the abscissa is pixel number, labeling the corresponding pixel position of each gray value. Gray scale values between vessels and ring artifacts were similar, with no statistically significant differences 

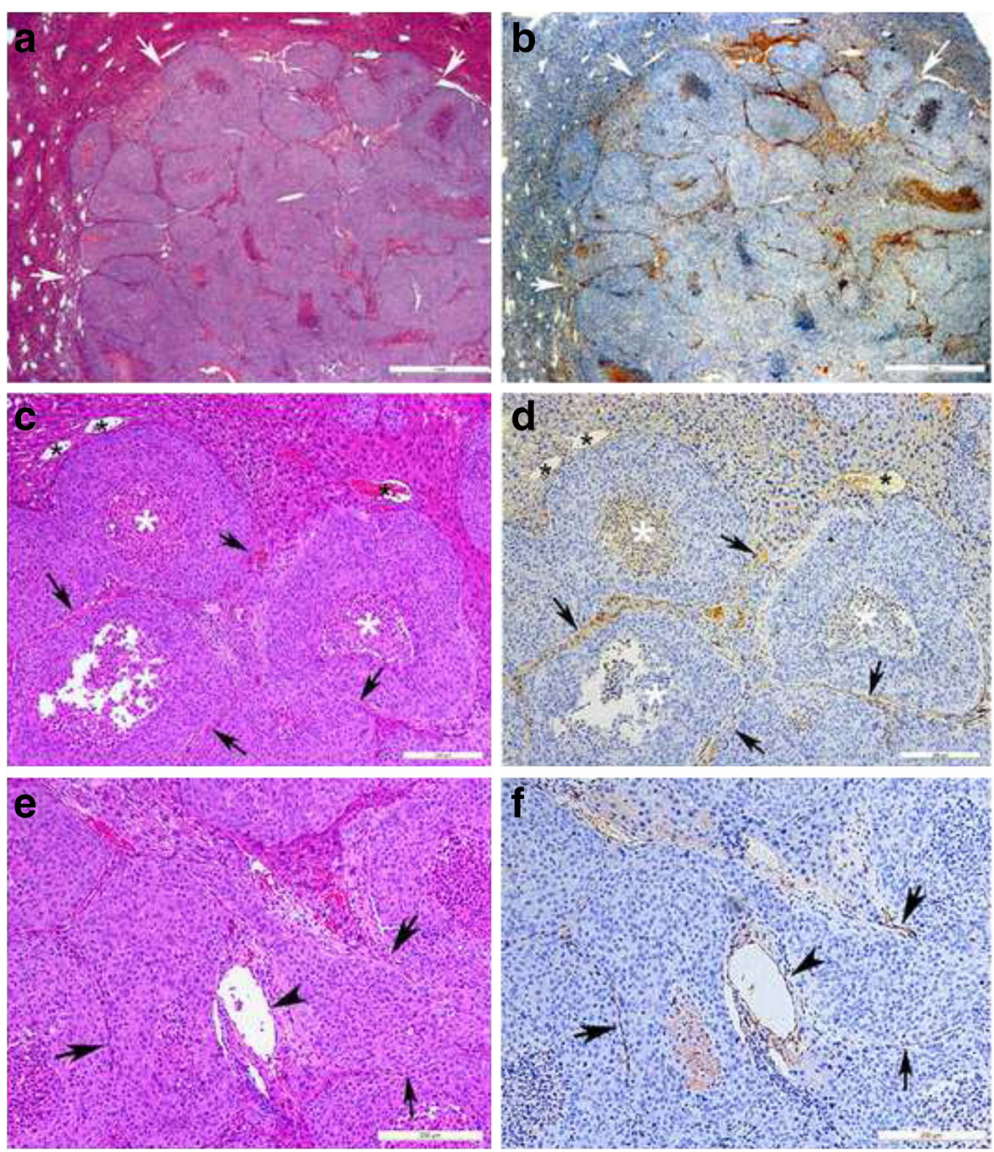

Fig. 7 Histopathological images of HCCLM3 orthotopic xenograft tumors at 2 weeks. Left: H\&E staining. Right: CD34 immunohistochemistry. a b Histopathological images of cancer tissues at week 2. Scale bar $=1 \mathrm{~mm}$. c d Histopathological images of cancer tissues at week 2. Bar $=200 \mu \mathrm{m}$. e f Histopathological images of cancer tissues at week 3. Bar $=200 \mu \mathrm{m}$. The boundaries between tumor and normal liver tissue could be shown clearly (a and $\mathbf{b}$, white arrow). CD34 expression was positive in the abundant normal hepatic sinusoid (c and $\mathbf{d}$, black asterisks) and tumor angiogenesis (c to $\mathbf{f}$, black arrows and arrow heads). The tumor edge or peripheral vasculature was compressed, having a shift in its curvature. The microvascular-rich regions were diffusely distributed at the edge of tumor nests. New vessels with different diameters were seen. The abnormal large vessels (e and $\mathbf{f}$, black arrow heads) and slit-like small vessels (c to $\mathbf{f}$, black arrows) coexisted. The characteristics of the vascular arrangements in $\mathrm{PCl}$ images were partly similar with those of histological sections. In addition, necrosis could be seen in the tumor nest (c and $\mathbf{d}$, white asterisks). The MVD of CD34 expression in tumors was determined by immunohistochemistry. Data are shown as median (Q25, Q75)

However, phase-contrast CT imaging is more challenging in tumor angiogenesis imaging and quantitative calculations than absorption-contrast CT. Indeed, phasecontrast CT suffers from ring artifacts: the gray value difference between the vessel and ring artifacts is small, rendering difficult the threshold setting of vascular segmentation in CT images and interfering with blood vessel identification. If the set threshold is too low, ring artifacts may be classified as vessels, thereby overevaluating the vessel density. If it is too high, actual vessel density will be underestimated. Future studies should be carry out to remove ring artifacts and improve the ability to identify vessels. During tumor growth, both necrotic tissue and tiny vessels were similarly gray. Therefore, it was difficult to distinguish between the two entities. Thus, vascular density in vessel segmentation may be overestimated. Finally, hand-drawing of the tumor region was subjective, as was the selection of the areas by different observers.

The present study had some limitations. First, a $9 \mu \mathrm{m}$ CCD detector was chosen considering sample size and thickness; therefore, the smallest vessel diameter detected was approximately $20 \mu \mathrm{m}$. Since the capillary diameter varies from a few to a hundred $\mu \mathrm{m}$, vessels of less than $20 \mu \mathrm{m}$ were not observed and tumor vascular density could be underestimated. Therefore, we will use higher-resolution detectors in future studies. Second, the $\mathrm{X}$-ray energy and object-to-detector distance were selected based on in vitro experiments. Finally, the imaging results were acquired from ethanol-dehydrated, formalin-fixed specimens. As a pioneer imaging technique, SR-based IL-XPCT, mainly limited by small beam size and 
high radiation dose, still needs further optimization to suit clinical application. Even in vivo imaging of neovascularization remains a challenging task. Therefore, the currently available studies mainly concentrate on in vitro specimen imaging to optimize the settings as well as to find the potential application field. In the future, provided that the problems related to technical facilities, imaging parameters and experimental model preparation are resolved, in vivo application of IL-XPCT is promising. Optionally, combining GB-PCI with conventional X-ray tube may be applicable in clinical conditions. Future studies should also examine bone samples from bone tumors or metastatic bone lesions using IL-XPCT, comparing the results with existing micro-CT data for such specimens.

\section{Conclusion}

In this study, we demonstrated that SR-based ILXPCT successfully acquires images of neovascularization in liver tumor xenografts. This technique can distinguish tumors from the normal liver tissue, and detect blood vessels as small as $20 \mu \mathrm{m}$ in diameter. In addition, quantitative analysis showed that vascular density increases first and then decreases gradually with tumor growth.

\section{Abbreviations}

CTA: Computed tomography angiography; DEl: Diffraction enhanced imaging; DSA: Digital subtraction angiography; GB-PCl: Grating-based phase-contrast X-ray imaging; IL-PCl: In-line phase contrast imaging; IL-XPCT: In-line X-ray phase contrast computed tomography; MRA: Magnetic resonance angiography; PCl: Phase-contrast imaging; SR: Synchrotron radiation; SRXTM: Synchrotron-based $\mathrm{X}$-ray tomographic microscopy; SSRF: Shanghai synchrotron radiation facility

\section{Acknowledgments}

We would like to thank the staff at the BL13W1 station of SSRF for help during the experiments.

\section{Funding}

This work was financially supported by the National Basic Research Program of China (973 Program 2010CB834305). The funder had role in covering the various costs related to this study, including materials, equipment, testing, processing, publication, transportation and labor.

\section{Availability of data and materials}

The dataset supporting the conclusions of this article is included within the article.

\section{Authors' contributions}

BLL, YQZ and SLC made substantial contributions to conception and design; BLL, YQZ, WZW, GHD, LC and HCS made substantial contributions to data acquisition, analysis and interpretation; BLL, YQZ and SLC were involved in drafting the manuscript or revising it critically for important intellectual content; all authors have given final approval of the version to be published.

\section{Competing interests}

The authors declare that they have no competing interests.

\section{Consent for publication}

Not applicable.

\section{Ethics approval}

All animal experiments were approved by the Animal Care and Use Committee of Zhongshan Hospital, and were conducted in accordance with all state regulations.

\section{Author details}

'Department of Nuclear Medicine, Zhongshan Hospital, Fudan University, No.180, Fenglin Road, Shanghai 200032, China. ${ }^{2}$ Shanghai Institute of Medical Imaging, Shanghai 200032, China. ${ }^{3}$ Liver Cancer Institute, Zhongshan Hospital, Fudan University, Shanghai 200032, China. ${ }^{4}$ Shanghai Institute of Applied Physics, Chinese Academy of Sciences, Shanghai 201800, China.

Received: 16 April 2016 Accepted: 18 January 2017

Published online: 25 January 2017

\section{References}

1. Hanahan D, Weinberg RA. Hallmarks of cancer: the next generation. Cell. 2011:144:646-74.

2. Villablanca JP, Rodriguez FJ, Stockman T, Dahliwal S, Omura M, Hazany S, et al. MDCT angiography for detection and quantification of small intracranial arteries: comparison with conventional catheter angiography. AJR Am J Roentgenol. 2007;188:593-602.

3. Wang $H$, Zheng LF, Feng $Y$, Xie $X Q$, Zhao JL, Wang XF, et al. A comparison of 3D-CTA and 4D-CE-MRA for the dynamic monitoring of angiogenesis in a rabbit VX2 tumor. Eur J Radiol. 2012;81:104-10.

4. Lundstrom U, Larsson DH, Burvall A, Takman PA, Scott L, Brismar H, et al. X-ray phase contrast for CO2 microangiography. Phys Med Biol. 2012;57:2603-17.

5. Nyangoga H, Mercier P, Libouban H, Basle MF, Chappard D. Threedimensional characterization of the vascular bed in bone metastasis of the rat by microcomputed tomography (MicroCT). PLoS One. 2011;6:e17336.

6. Yamashita T. Evaluation of the microangioarchitecture of tumors by use of monochromatic x-rays. Invest Radiol. 2001;36:713-20

7. Tokiya R, Umetani K, Imai S, Yamashita T, Hiratsuka J, Imajo Y. Observation of microvasculatures in athymic nude rat transplanted tumor using synchrotron radiation microangiography system. Acad Radiol. 2004;1 1:1039-46.

8. Liu X, Zhao J, Sun J, Gu X, Xiao T, Liu P, et al. Lung cancer and angiogenesis imaging using synchrotron radiation. Phys Med Biol. 2010;55:2399-409.

9. Zhou SA, Brahme A. Development of phase-contrast X-ray imaging techniques and potential medical applications. Phys Med. 2008;24:129-48.

10. Bonse U, Hart M. An X-ray interferometer. Appl Phys Lett. 1965:6:155-6.

11. Chapman D, Thomlinson W, Johnston RE, Washburn D, Pisano E, Gmur N, et al. Diffraction enhanced X-ray imaging. Phys Med Biol. 1997;42:2015-25.

12. Zhang $X$, Yang XR, Chen Y, Li HQ, Li RM, Yuan QX, et al. Visualising liver fibrosis by phase-contrast $X$-ray imaging in common bile duct ligated mice. Eur Radiol. 2013;23:417-23.

13. Sztrokay A, Herzen J, Auweter SD, Liebhardt S, Mayr D, Willner M, et al. Assessment of grating-based $\mathrm{X}$-ray phase-contrast $\mathrm{CT}$ for differentiation of invasive ductal carcinoma and ductal carcinoma in situ in an experimental ex vivo set-up. Eur Radiol. 2013;23:381-7.

14. Snigirev A, Snigireva I, Kohn V, Kuznetsov S, Schelokov I. On the possibilities of $\mathrm{x}$-ray phase contrast microimaging by coherent high-energy synchrotron radiation. Rev Sci Instrum. 1995;66:5486-92.

15. Lundstrom U, Larsson DH, Burvall A, Scott L, Westermark UK, Wilhelm $M_{\text {, }}$ et al. X-ray phase-contrast $\mathrm{CO}(2)$ angiography for sub-10 mum vessel imaging. Phys Med Biol. 2012;57:7431-41.

16. Stampanoni M, Groso A, Isenegger A, Mikuljan G, Chen Q, Bertrand A et al., editors. Trends in synchrotron-based tomographic imaging: the SLS experience. SPIE Optics + Photonics; 2006: International Society for Optics and Photonics.

17. Beheshti A, Pinzer BR, McDonald JT, Stampanoni M, Hlatky L. Early tumor development captured through nondestructive, high resolution differential phase contrast X-ray imaging. Radiat Res. 2013;180:448-54.

18. Hu JZ, Wu TD, Zeng L, Liu HQ, He Y, Du GH, et al. Visualization of microvasculature by $x$-ray in-line phase contrast imaging in rat spinal cord. Phys Med Biol. 2012;57:N55-63.

19. Tang R, Chai WM, Ying W, Yang GY, Xie H, Liu HQ, et al. Anti-VEGFR2conjugated PLGA microspheres as an $\mathrm{x}$-ray phase contrast agent for assessing the VEGFR2 expression. Phys Med Biol. 2012;57:3051-63.

20. Yang BW, Liang Y, Xia JL, Sun HC, Wang L, Zhang JB, et al. Biological characteristics of fluorescent protein-expressing human hepatocellular carcinoma xenograft model in nude mice. Eur J Gastroenterol Hepatol. 2008:20:1077-84.

21. Zhang X, Liu XS, Yang XR, Chen SL, Zhu PP, Yuan QX. Mouse blood vessel imaging by in-line $x$-ray phase-contrast imaging. Phys Med Biol. 2008;53:5735-43. 
22. Draijer M, Hondebrink E, van Leeuwen T, Steenbergen W. Review of laser speckle contrast techniques for visualizing tissue perfusion. Lasers Med Sci. 2009;24:639-51.

23. Savai R, Langheinrich AC, Schermuly RT, Pullamsetti SS, Dumitrascu R, Traupe $\mathrm{H}$, et al. Evaluation of angiogenesis using micro-computed tomography in a xenograft mouse model of lung cancer. Neoplasia. 2009;11:48-56.

24. Weidner N, Semple JP, Welch WR, Folkman J. Tumor angiogenesis and metastasis—correlation in invasive breast carcinoma. N Engl J Med. 1991;324:1-8.

25. Cai W, Gambhir SS, Chen X. Chapter 7. Molecular imaging of tumor vasculature. Methods Enzymol. 2008;445:141-76.

26. McDonald DM, Choyke PL. Imaging of angiogenesis: from microscope to clinic. Nat Med. 2003;9:713-25.

27. Duan J, Hu C, Chen H. High-resolution micro-CT for morphologic and quantitative assessment of the sinusoid in human cavernous hemangioma of the liver. PLoS One. 2013;8:e53507.

28. Zhang X, Yang XR, Chen Y, Li HQ, Liu WY, Yuan QX, et al. Diffractionenhanced radiography of various mouse organs. AJR Am J Roentgenol. 2010;195:545-9.

29. Suortti P, Thomlinson W. Medical applications of synchrotron radiation. Phys Med Biol. 2003;48:R1.

30. Laperle CM, Hamilton TJ, Wintermeyer P, Walker EJ, Shi D, Anastasio MA, et al. Low density contrast agents for $x$-ray phase contrast imaging: the use of ambient air for $x$-ray angiography of excised murine liver tissue. Phys Med Biol. 2008:53:6911-23.

31. Zhang D, Yan X, Zhang X, Liu C, Dang R, Xiao T, et al. Synchrotron radiation phase-contrast $X$-ray CT imaging of acupuncture points. Anal Bioanal Chem. 2011:401:803-8.

32. Zhang MQ, Sun DN, Xie YY, Peng GY, Xia J, Long HY, et al. Threedimensional visualization of rat brain microvasculature following permanent focal ischaemia by synchrotron radiation. Br J Radiol. 2014;87:20130670.

33. Fu J, Tan R. In-line phase contrast micro-CT reconstruction for biomedical specimens. Biomed Mater Eng. 2014;24:431-7.

\section{Submit your next manuscript to BioMed Central and we will help you at every step:}

- We accept pre-submission inquiries

- Our selector tool helps you to find the most relevant journal

- We provide round the clock customer support

- Convenient online submission

- Thorough peer review

- Inclusion in PubMed and all major indexing services

- Maximum visibility for your research

Submit your manuscript at www.biomedcentral.com/submit 